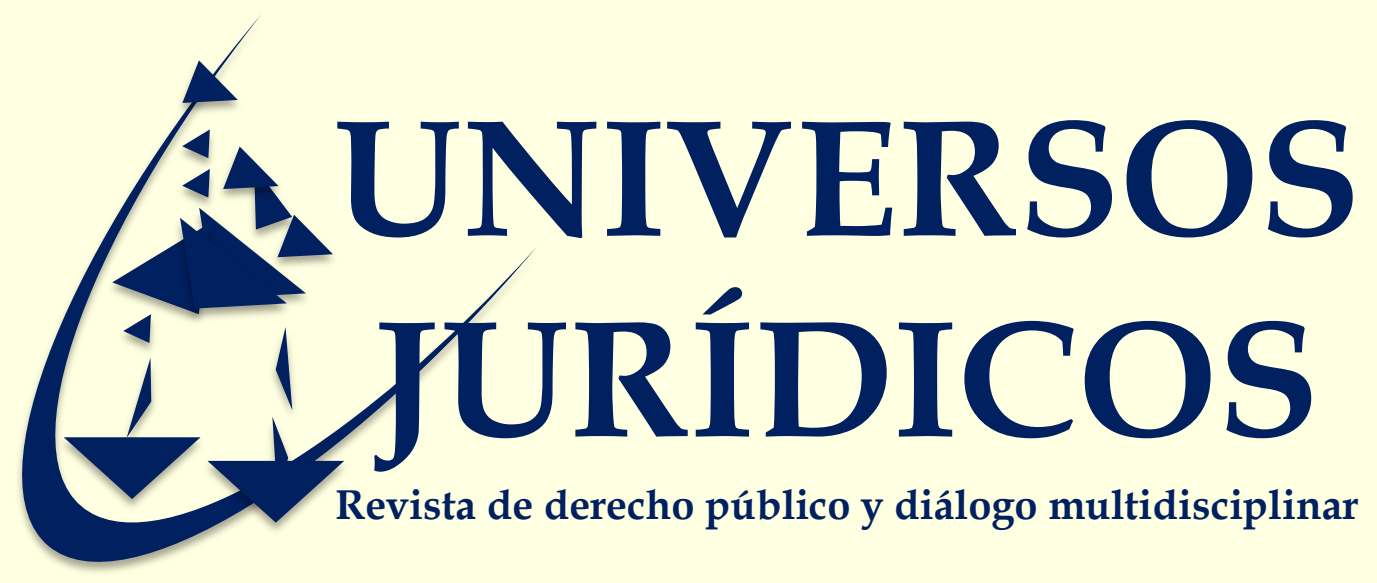

\title{
Aproximación a la metodología de la investigación jurídica
}

Approach to the methodology of legal research

Eduardo de Jesús Castellanos Hernández 1

El conocimiento científico y la racionalidad humana

que éste produce son, creo, siempre falibles,

están siempre sujetos a error.

Pero también son, creo, orgullo de la humanidad

Karl Popper

UNIVERSOS JURÍDICOS. Revista de derecho público y diálogo multidisciplinar. Año 8, No. 15, noviembre 2020-abril 2021, ISSN 2007-9125

Cómo citar este artículo en formato APA Castellanos, E. (2020). Aproximación a la metodología de la investigación jurídica. Universos Jurídicos, 58-83.

Fecha de recepción: 21 de mayo de 2020

Fecha de aceptación: 12 septiembre de 2020

\begin{abstract}
1. Investigador Nacional, Nivel I, adscrito al Instituto Interdisciplinario de Investigaciones de la Universidad de Xalapa; miembro del Registro CONACYT de Evaluadores Acreditados. Área 5, Sociales y Económicas; licenciado en Derecho, UNAM; maestro en Administración de Empresas, UAEMéx; doctorado en Estudios Políticos, Universidad de París; Especialidad en Justicia Electoral, TEPJF; Posdoctorado en Control Parlamentario y Políticas Públicas, Universidad de Alcalá; Posdoctorado en Regímenes Políticos Comparados, Universidad de Colorado, Colorado Springs; doctorante en Derecho, Instituto Internacional del Derecho y del Estado, Ciudad de México.

eduardocastellanosh@gmail.com
\end{abstract}




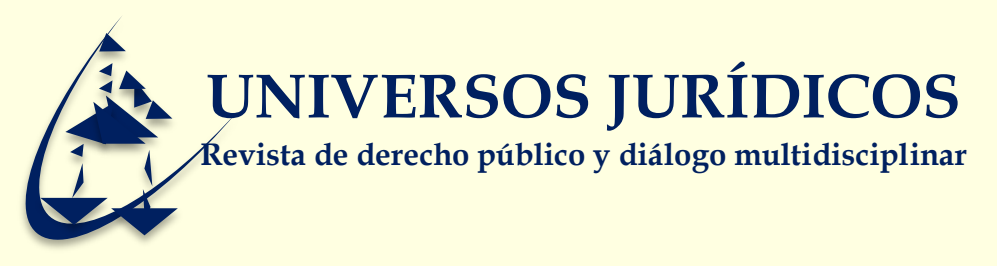

Sumario: I. Introducción. II. El método de la investigación.

III. La metodología de la investigación jurídica. IV. Técnicas e instrumentos de recolección de datos. V. El diseño de la investigación jurídica. VI. El reporte de la investigación. VII. Conclusiones. VII. Fuentes de consulta.

Resumen: A partir de la convicción de que todos los productos jurídicos en esencia son o deberían ser reportes de una investigación llevada a cabo con el rigor de la metodología de investigación de las ciencias sociales, se hace un recorrido por algunos de los conceptos centrales de la teoría del conocimiento, su método, su metodología y sus técnicas e instrumentos de recolección de datos, aplicados todos a la investigación jurídica.

Palabras clave: método, metodología, investigación jurídica, reporte de investigación, productos jurídicos.

Abstract: Based on the conviction that all legal products in essence are or should be reports of research carried out with the rigor of the research methodology of the social sciences, it takes a tour of some of the central concepts of knowledge theory, its method, its methodology and its data collection techniques and instruments, all applied to legal research. 


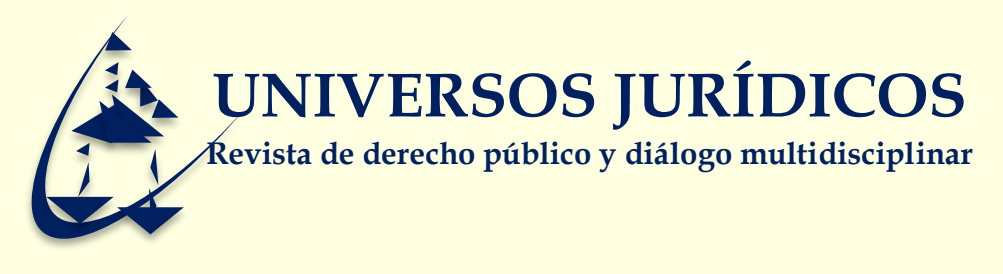

Keywords: method, methodology, legal research, investigation report, legal products.

\section{Introducción}

Con frecuencia escuchamos hablar de la Ciencia Jurídica, lo que impulsa a los abogados y a los estudiantes de derecho a pensar que su disciplina profesional no solo tiene un carácter científico en sí misma, sino que, además, es prácticamente una disciplina autónoma. Esto sucede, tal vez, porque habitualmente en los planes de estudio de la licenciatura en Derecho no existe la asignatura Metodología de la Investigación; en parte, porque se da por descontado que dichos temas fueron estudiados en la enseñanza media superior, pero también porque por diversas razones ha desaparecido prácticamente la obligación de presentar y sustentar una memoria de investigación (tesis) para la obtención del título de licenciado en Derecho. Sin embargo, esto no impide afirmar que la metodología de la investigación jurídica deriva y, por lo tanto, está directamente vinculada al estudio del método de la ciencia y de la metodología de la investigación en ciencias sociales.

Es por ello que en los distintos niveles de posgrado en Derecho necesariamente aparece el estudio de la Metodología de la Investigación, aunque con denominaciones que aparentemente ocultan este contenido bajo otras designaciones como Seminario de Investigación, por ejemplo, cuyo contenido o punto de partida inevitablemente es la metodología de investigación en ciencias sociales.

Esta aparición de la metodología en los niveles de posgrado no se explica más que por la necesidad de afirmar la cientificidad de los estudios jurídicos, pues 


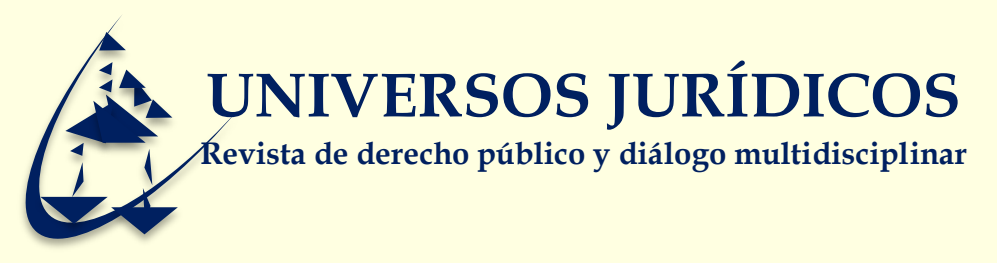

la redacción de tesis de grado prácticamente ha desaparecido en el nivel licenciatura -como ya lo he señalado-, aunque también en los niveles de maestría -en el de especialidad, me parece, nunca ha existido-. Al paso que vamos poco falta para que ni siquiera para obtener el grado de doctor en Derecho sea necesario presentar un trabajo de investigación.

Lo que resulta paradójico de esta ausencia de preparación a los estudiantes de Derecho en la redacción de reportes de investigación jurídica, es que los productos de los diferentes procedimientos y procesos propios de la disciplina jurídica y, más aún, de la profesión de abogado, además de requerir de una metodología de investigación jurídica -predominantemente documental, aunque en ocasiones necesariamente de campo--, obligatoriamente culminan con un reporte de investigación que adquiere diversos nombres totalmente alejados del que hasta aquí he comentado -tesis de grado o posgrado-, pero que sin la menor duda son reportes de investigación, por ejemplo: contrato, demanda, contestación de demanda, sentencia, iniciativa de ley o ley y muchos más (juicio político, declaración de procedencia, dictamen legislativo, artículo jurídico, libro jurídico, criterio jurisprudencial, peritaje, etc.). Puesto que ninguno de estos productos jurídicos surge de la nada o es invención propia de sus autores, sino resultado de una investigación jurídica sometida a los mismos cánones de la metodología de la investigación en ciencias sociales para ser viable.

Es por ello que de manera lo más breve posible me propongo recordar algunos conceptos bastante conocidos por los investigadores sociales en otras disciplinas -aunque no siempre por los estudiantes de Derecho-, algunos de cuyos instrumentos de trabajo son habitualmente utilizados en las investigaciones jurídicas que culminan con los reportes de investigación metodológica -aunque 


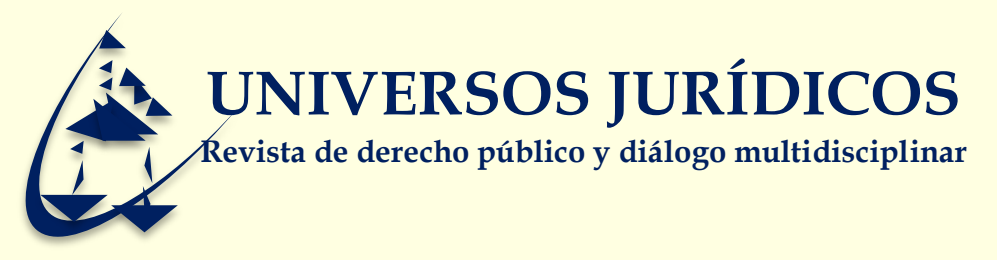

frecuentemente sin metodología, o bien aprendida ésta a partir del método de prueba y error o del de imitación reiterada o el de francamente llenar "machotes"que en la práctica jurídica se denominan de tan diversas formas, como ya se ha dicho.

Una mención final para introducir lo que sigue es que habiendo sido invitado a impartir la materia Seminario de Investigación como asignatura común a las maestrías en Derecho Corporativo, Derecho Fiscal y Amparo, que se cursan en el Plantel Tlalnepantla de la Universidad Insurgentes, me vi en la necesidad de revisar la bibliografía estudiada en cursos recientes y lejanos, tanto cursados como alumno como impartidos como profesor -desde aquellos años distantes en que fui profesor de Temas de Ciencias Sociales en el Colegio de Bachilleres en la hoy Ciudad de México o alumno del doctorado en Estudios Políticos en la Universidad de París o, más recientemente, apenas durante los dos años pasados, como alumno del doctorado en Derecho en la Ciudad de México-.

La bibliografía consultada para la redacción de este artículo permitirá al lector interesado en su contenido adentrarse en los diversos aspectos del asunto planteado. Pero, sobre todo, espero que le auxilie y facilite para realizar investigaciones jurídicas cuyo reporte final cubra los requisitos metodológicos sustanciales de una investigación propia de las ciencias sociales, y sea presentado mediante un documento redactado de manera coherente, entendible y que cumpla el propósito para el cual fue hecho.

\section{El método de la investigación}

Mario Bunge (Bunge, 1973, 8 y sigs.) establece una primera gran división de las ciencias en formales (lógica, matemáticas) y fácticas (naturales y sociales). 


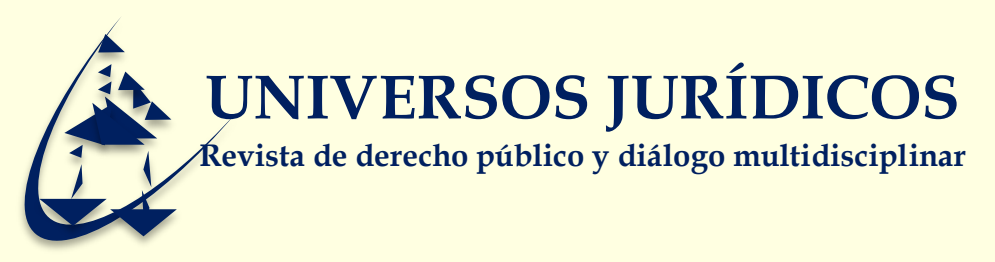

Precisa que las ciencias formales demuestran o prueban, en tanto que las ciencias fácticas verifican (confirman o disconfirman) hipótesis que en su mayoría son provisionales.

Señala también que las principales características de las ciencias fácticas son: que trascienden los hechos (descartan hechos, producen nuevos hechos y los explican); su conocimiento es racional; objetivo; analítico (aborda problemas y trata de descomponerlo todo en elementos); especializado; claro y preciso; comunicable; verificable (las proposiciones generales verificables son hipótesis científicas); metódico; sistemático; general; legal; explicativo; predictivo; abierto; útil.

El Diccionario de la Lengua Española de la Real Academia Española (RAE, 1992, 1366) define la palabra método de la siguiente forma: "Modo de decir o hacer con orden una cosa. 2. Modo de obrar o proceder; hábito o costumbre que cada uno tiene y observa. 3. Procedimiento que se sigue en las ciencias para hallar la verdad y enseñarla. Puede ser analítico o sintético. 4. Obra que enseña los elementos de una ciencia o arte". En tanto que la palabra metodología la define como "Ciencia del método. 2. Conjunto de métodos que se siguen en una investigación científica o en una exposición doctrinal".

Según Bunge, en la obra citada (Bunge, 1973, 89-92), el método científico es a grandes líneas el siguiente: 1. Planteo del problema (reconocimiento de los hechos, descubrimiento del problema y formulación del problema); 2.Construcción de un modelo teórico (selección de los factores pertinentes, invención de las hipótesis centrales y de las suposiciones auxiliares, traducción matemática); 3. Deducción de consecuencias particulares (búsqueda de soportes racionales, 


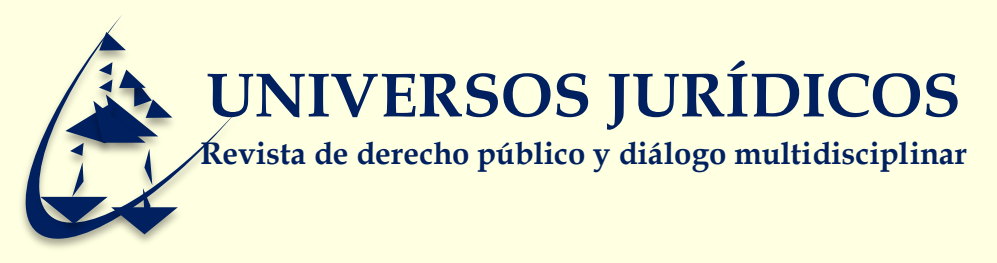

búsqueda de soportes empíricos); 4. Prueba de la hipótesis (diseño de la prueba, ejecución de la prueba, elaboración de los datos, inferencia de la conclusión); 5. Introducción de las conclusiones en la teoría (comparación de las conclusiones con las predicciones, reajuste del modelo, sugerencias acerca del trabajo ulterior).

En una obra posterior (Bunge, 2015, 39-40), Mario Bunge presenta una formulación actualizada del método científico con las siguientes etapas: 1 . Descubrimiento del problema; 2. Planteo preciso del problema; 3. Búsqueda de conocimientos 0 instrumentos relevantes; 4 . Tentativa de solución del problema con ayuda de los medios identificados; 5. Invención de nuevas ideas (hipótesis, teorías o técnicas) o producción de nuevos datos empíricos que prometan resolver el problema; 6. Obtención de una solución (exacta o aproximada); 7. Investigación de las consecuencias de la solución obtenida; 8. Puesta a prueba (contrastación) de la solución; 9. Corrección de las hipótesis, teorías, procedimientos o datos empleados en la obtención de la solución incorrecta.

Max Weber (Weber, 1985, 70) se refiere a la falibilidad del conocimiento científico -de la misma manera y con igual optimismo que lo hace Karl Popper (Popper, 2005, 11) en la cita que aparece como epígrafe al inicio de este artículo-: "Sabemos muy bien que lo que hayamos producido se habrá vuelto arcaico dentro de diez, veinte o cincuenta años. Tal es el destino y el sentido del trabajo científico". Como también afirma, a su manera, Gastón Bachelard (Bachelard, 2013, 297): "En la obra de la ciencia sólo puede amarse aquello que se destruye, sólo puede continuarse el pasado negándolo, sólo puede venerarse al maestro contradiciéndolo". 


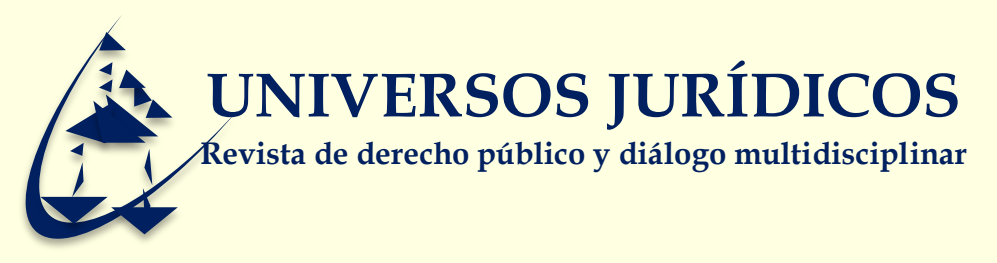

La ciencia es una fórmula más, aunque no la única, en la búsqueda de la verdad. Michel Foucault (Foucault, 2017, 46) afirma que "La modalidad técnica del decir veraz se organiza en mucho mayor medida alrededor de la ciencia que de la enseñanza, o, en todo caso, alrededor de un complejo constituido por las instituciones de ciencia e investigación y las instituciones de enseñanza."

Para Nicola Abbagnano (Abbagnano, 2000, 1130) la verdad es la validez 0 la eficacia de los procedimientos cognoscitivos. Distingue al efecto cinco conceptos fundamentales de la verdad: 1. Como correspondencia o relación; 2. Como revelación; 3. Como conformidad a una regla; 4. Como coherencia, y 5. Como utilidad.

En un libro reciente, Julian Baggini (Baggini, 2018, 7) propone la siguiente clasificación de los distintos tipos de verdad: 1. Verdades eternas (o religiosas), especiales por no ser del tipo común y empírico pues propugnan distintos fundamentos de verdad; 2 . Verdades de autoridad, puesto que toda cultura acepta a algunas personas como autoridad, aunque sea difícil establecer el equilibrio correcto entre nuestro propio juicio y el testimonio del experto; 3. Verdades esotéricas, pues la idea de que algunas verdades permanecen ocultas o son "esotéricas" es tan antigua como la civilización, frente a lo cual recomienda ser adecuadamente escéptico sin ser indiscriminadamente cínico para mantenerse como un pensador crítico; 4. Verdades razonadas, frente a las cuales hay que tener en cuenta que la razón es una herramienta imperfecta con usuarios imperfectos; 5. Verdades empíricas, demasiado modestas para afirmar una certeza absoluta toda vez que las pruebas rara vez consiguen una única, decisiva y emancipadora ruptura con el error; 6 . Verdades creativas, en las que si bien requieren de imaginación ésta no es suficiente para crear nuevas verdades; 7 . 


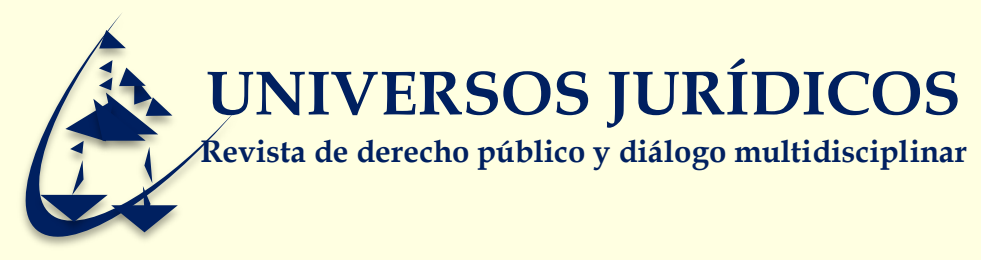

Verdades relativas, toda vez que hay más de una forma de describir el mundo, más de una forma de asignar valor e importancia a las cosas; 8. Verdades poderosas, en las que a pesar de la frecuente apropiación de la verdad por parte del poder, hay que tener en cuenta que la verdad es más que poder por lo que es posible decir la verdad al poder; 9. Verdades morales, en donde hay que considerar que nuestros juicios morales solo estarán bien fundamentados cuando concuerden con los hechos tanto de la naturaleza humana como del mundo; 10. Verdades holísticas, pues las verdades no se mantienen en pie o caen de forma independiente, sino que se sostienen sobre una red de otras verdades, que se apoyan mutuamente.

Hoy, cuando es frecuente escuchar hablar de la posverdad -la mentira disfrazada de verdad-, es conveniente recordar que Baggini concluye su taxonomía de la verdad refiriéndose a las verdades futuras afirmando que "Si deseamos conocer la verdad, podría asumirse que lo más necesario es un método de investigación o un conjunto de reglas para establecer hechos" (Baggini, 2018, p. 87). La vinculación entre las verdades futuras, el método y la metodología poco a poco resulta evidente.

\section{La metodología de la investigación jurídica}

Por su importancia, reitero dos cosas que ya he señalado. La primera es que la metodología de la investigación jurídica no es ajena, sino que es la misma metodología que se aplica en las demás ciencias sociales, toda vez que se trata de un único camino -el método científico- para encontrar la verdad científica. Es por ello que acudiré a desarrollar este acápite con el auxilio de autores de metodología de la investigación en ciencias sociales. La segunda es que los reportes de investigación jurídica pueden adquirir nombres muy variados pero 


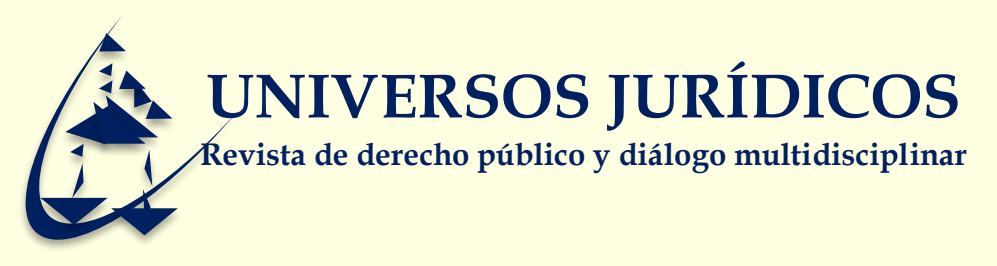

que, finalmente, son el resultado de una investigación -no solamente documental o libresca- que conduce a una verdad jurídica -pretendidamente científica-, que pueden tener cualquiera de los siguientes nombres: contrato, demanda, contestación de la demanda, sentencia, sentencia, iniciativa de ley, dictamen, ley, juicio político, declaración de procedencia o cualquier otro propio de nuestra disciplina y su operación cotidiana en tribunales, cámaras legislativas, despachos jurídicos u oficinas administrativas.

Hemos visto ya que todas las ciencias, formales o factuales, culminan o se expresan mediante una legalidad o normatividad -las reglas del silogismo, la ley de la gravitación universal o las leyes de la evolución-. Puesto que el Derecho es ciencia social, tiene su propia legalidad o reglas indispensables en el curso de una investigación jurídica -que generan otra legalidad-, como veremos en el siguiente acápite.

Pero toda vez que como ciencia social lo que busca el Derecho es, a partir del estudio de la realidad social, culminar con un contrato, una norma o resolución administrativa, sentencia judicial o resolución legislativa (ley, decreto, acuerdo, punto de acuerdo) cuya congruencia con la realidad social de la que surge sea óptima; la consistencia metodológica para llegar a ese resultado es esencial.

Jorge Padua (Padua, 1996, p. 30-31) señala que "La investigación científica tiene como sus objetivos teóricos más generales, dar respuestas inteligibles, confiables y válidas, a preguntas específicas o problemas de investigación". Señala, al efecto, que las respuestas tratan de responder acerca del qué o cómo, dónde, cuándo, de dónde y por qué, sin que se trate de responder todas las interrogantes pues, en ocasiones, solo se trate de contestar 


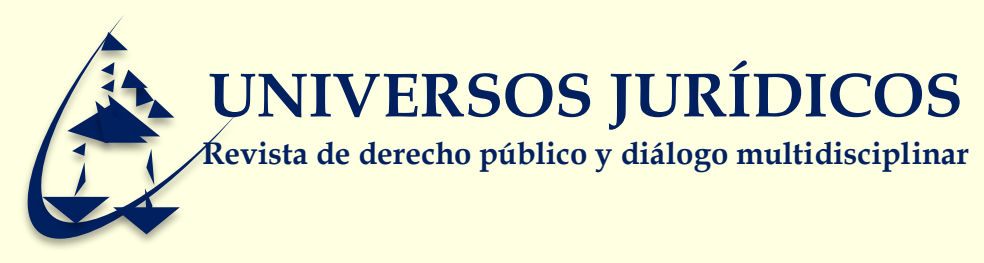

algunas de ellas. De ahí que, agrega, "La bibliografía especializada acostumbra diferenciar los estudios o diseños de investigación -según el tipo de pregunta que el investigador plantee- en estudios exploratorios, descriptivos y explicativos.

Afirma Felipe Pardinas (Pardinas, 1973, p. 10) que "El primer paso para un estudio empírico es la observación de los fenómenos que estudia una ciencia. Cada ciencia tiene como primer carácter distintivo el área de fenómenos a cuyo estudio está dedicada." En consecuencia, agrega, "El método de trabajo científico comienza pues con la observación de un área particular de fenómenos que interesa, por razones válidas, al investigador" (Pardinas, 1973, p. 11) -afirmación sujeta de inmediato a cuestionamiento al iniciar el siguiente acápite-.

Propone, en consecuencia, lo siguiente: formulación de un problema (rastrear problemas o formular preguntas); hipótesis (respuesta tentativa a la pregunta o al problema planteado); diseño de la prueba (procedimientos de comprobación o disprobación de la o las hipótesis); conclusiones o resultados y presentación de resultados.

La metodología tiene como propósito la descripción, explicación y predicción de las conductas de los fenómenos en estudio, insisto. Para dicho estudio contamos o acudimos a tres tipos de conocimiento: conocimientos populares, conocimientos de divulgación y conocimientos científicos. El estudio científico consta de dos elementos básicos: la teoría y el método. La metodología es el estudio crítico del método.

Carlos I. Muñoz Rocha (Muñoz, 2016, p. 104), por su parte, propone las siguientes etapas del proceso de investigación: 1. Reflexión o lógica; 2. 


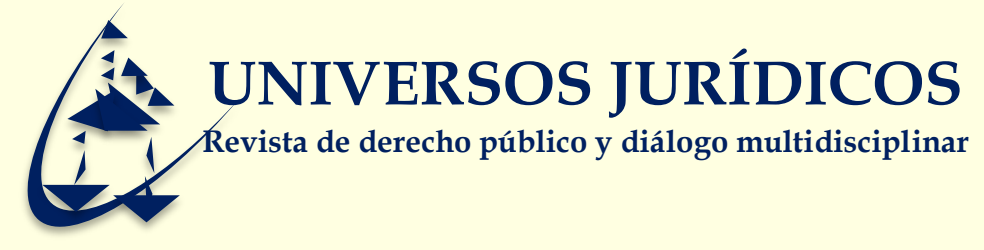

Planeación y diseño o metodológica; 3. Desarrollo y ejecución o técnica; 4. De resultados o síntesis.

La etapa de investigación comprende los pasos siguientes: 1. Área de investigación; 2. Selección del tema, idea o proyecto de investigación; 3. Planteamiento del problema; 4. Delimitación o alcance del problema; 5. Construcción del marco teórico. En tanto que la etapa de planeación y diseño o metodológica incluye: 1. Diseño de la investigación; 2. Hipótesis de trabajo; 3. Indicadores.

Por su parte, a la etapa de desarrollo y ejecución o técnica le atribuye los pasos siguientes: 1. Instrumentos de recolección de datos; 2. Técnicas de recolección de datos; 3 . Los datos o información; 4. Procesamiento de la información; 5. Análisis de los datos o información. Para considerar, finalmente en la etapa de resultados o síntesis lo siguiente: 1. La elaboración de síntesis y conclusiones, y 2. La redacción del informe de resultados.

Por paradójico que parezca, la verdad científica -como ha quedado dicho desde el epígrafe- es provisional. La fuerza explicativa de las causas y las interrelaciones de un fenómeno estudiado a través de una hipótesis o de una teoría puede cambiar o desaparecer con la llegada de nuevas hipótesis y teorías. Los autores llaman a eso de diferentes maneras: revolución científica, ruptura epistemológica o golpe de Estado teórico.

Thomas S. Kuhn (Khun, 2015, p. 230) señala "consideramos como revoluciones científicas aquellos episodios de desarrollo no acumulativo en los que un paradigma (Khun, 2015, p. 161) antiguo se ve sustituido en todo o en parte por otro nuevo incompatible con él". En tanto que Pierre Bourdieu et al (Bourdieu, 


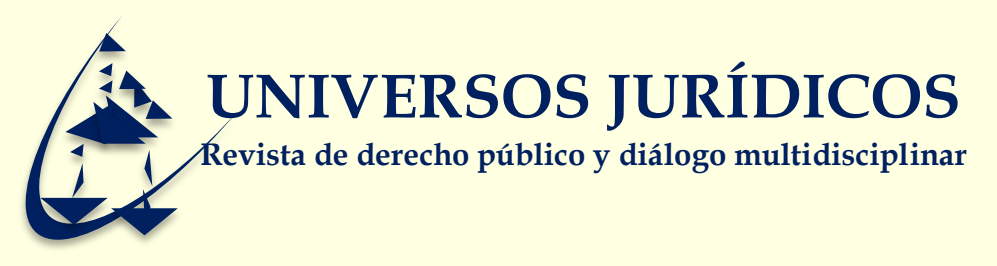

$2016,105)$ sostienen que "por el hecho de que los límites entre el saber común y la ciencia son, en sociología, más imprecisos que en cualquier otra disciplina, la necesidad de la ruptura epistemológica se impone con particular urgencia”.

\section{Técnicas e instrumentos de recolección de datos}

Karl Popper (Popper, 2005, 182-183) advierte que "No se puede empezar por la observación: es menester saber primero qué observar. Esto es, es menester comenzar por un problema. Además, no existe observación exenta de interpretación. Todas las observaciones son interpretadas a la luz de las teorías" (a algunas teorías en materia jurídica me referiré en el siguiente acápite). Por su parte, Pierre Bourdieu et al (Bachelard, 2013, p. 92) enfatizan: "La más elemental de las operaciones, la observación, que describe el positivismo como un registro tanto más fiel cuanto menos supuestos teóricos implica, se hace cada vez más científica en tanto los principios teóricos que la sostienen son más conscientes y sistemáticos". Puesto que, como advierte Gastón Bachelard (Bachelard, 2017, 27), "En la formación de un espíritu científico, el primer obstáculo es la experiencia básica, es la experiencia colocada por delante y por encima de la crítica, que, ésta sí, es necesariamente un elemento integrante del espíritu científico".

El área de estudio de las ciencias sociales son las conductas humanas y su observación se realiza a través de cuatro tipos principales, comunes a todas las ciencias sociales, que son: observaciones o datos documentales, observaciones de campo que pueden ser de monumentos o de campo, y las observaciones de laboratorio. 


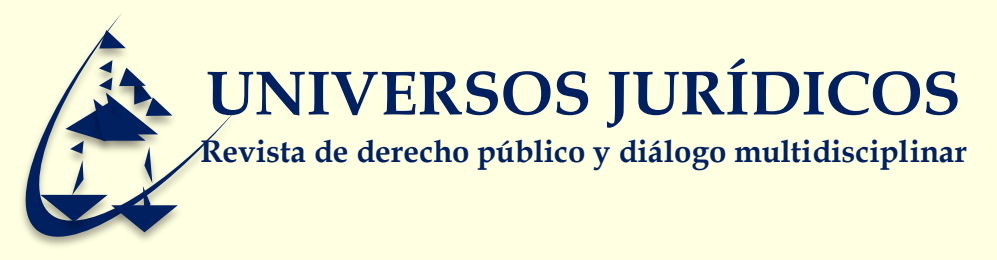

En la ciencia jurídica las observaciones son fundamentalmente documentales y de campo, pero con el apoyo de otras ciencias factuales, no necesariamente solo ciencias sociales, puede aprovechar para sus conclusiones de las otras formas de observación que incluyan métodos cuantitativos y de laboratorio, es decir, de la evaluación cuantitativa y cualitativa de la materia, las conductas y los hechos. , Un ejemplo de lo anterior son los trabajos de Báez y Jongitud $(2013,2014)$ que implican un manejo estadístico en investigación jurídica.

Por su parte, la observación de conductas en el campo puede ser participante ${ }^{1} \mathrm{o}$ no-participante mediante una observación pasiva y no comprometida con los resultados propios del proceso social observado, pero sí comprometida desde luego con la confirmación o no de las hipótesis que los expliquen.

Entre las técnicas preliminares a las observaciones de campo se tienen: muestreo, cuestionarios, entrevistas, investigación de actitudes, encuestas de opinión y la observación experimental.

Pardinas -al igual que todos los demás autores- señala que "La multitud de datos y observaciones que recoge cualquier científico no tienen valor mientras no puedan ser ordenados en distintos tipos de conducta, reuniendo en un tipo los fenómenos u observaciones que poseen características estructurales 0 conductistas semejantes" (Pardinas, 1973, p. 115). Entre tanto, es oportuno tener en cuenta que dichos resultados pueden ser presentados mediante cuadros de datos y tablas de frecuencias que permitan o faciliten operaciones comparativas.

\footnotetext{
${ }^{1}$ Por ejemplo, participar como actor en procesos político electorales para observar su realización y sacar conclusiones o en procesos de selección de funcionarios para el mismo propósito. 


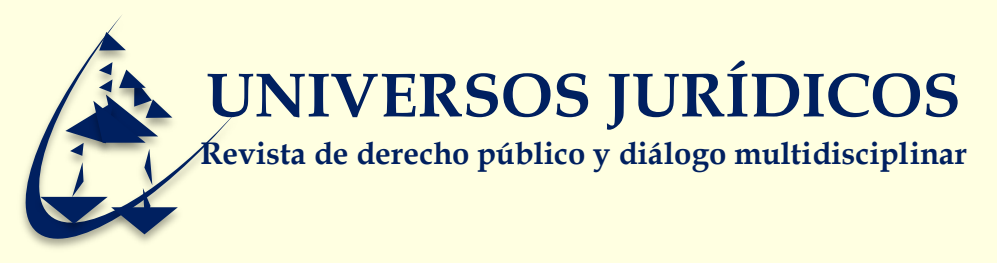

Si bien la hipótesis pretende resolver o explicar un problema, es conveniente recordar que podemos tener hipótesis alternativas, hipótesis nulas e hipótesis de trabajo.

La presentación de los resultados de la investigación, según el autor ahora en cita, incluye las partes siguientes: 1. Prólogo; 2. Contenido o índice general; 3. Introducción; 4. Diseño de la prueba; 5. Codificación, procesamiento y análisis de los datos; 6. Conclusiones; 7. Tablas o cuadros; 8. Notas al texto; 9. Bibliografía, 10. Índice alfabético de materia y de nombres. Es frecuente que cada institución tenga su propia metodología o forma de presentar sus resultados, sea en forma de libro, artículo u otra, por lo que el investigador deberá ajustarse a la práctica que le corresponda.

Respecto a las dificultades para seleccionar los temas o ideas de investigación, Muñoz Rocha identifica tres grupos. El primero, relacionado con los propios estudiantes; el segundo, con las instituciones educativas y, el tercero, con los tutores.

Por cuanto a los estudiantes enumera las siguientes dificultades (Muñoz, 2016, p. 118):

- Ausencia de hábitos de lectura, incluso en estudiantes de doctorado.

- Inexperiencia en actividades de investigación.

- Desconocimiento de técnicas de investigación documental.

- Incompetencia para buscar información.

- Falta de dominio de la ortografía española.

- Carencia de disciplina para el estudio y la investigación (quizá lo más grave de lo enunciado anteriormente). 


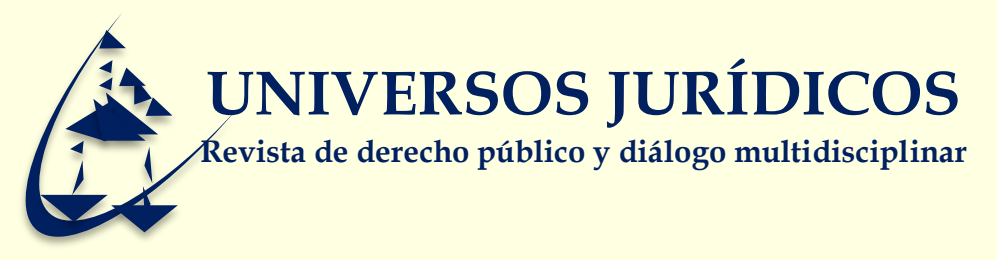

Se trata de dificultades que sin duda tendrán un alto costo para el estudiante de licenciatura o posgrado en el desarrollo de su vida profesional. Por eso es de felicitarse que se mantenga la asignatura Seminario de Investigación, o cualquier otro nombre que se le asigne, siempre que esté destinada a crear y afirmar la disciplina metodológica de investigación en los profesionales del Derecho.

Una vez formuladas estas consideraciones de carácter metodológico para la investigación en las ciencias sociales, aplicables desde luego a la ciencia jurídica, paso ahora a los aspectos propios de la investigación en materia jurídica de manera más específica.

\section{EL DISEÑO DE LA INVESTIGACIÓN JURÍDICA}

El diseño de la investigación jurídica en México hoy tiene que partir de la constatación de los cambios ocurridos en diversos espacios de la disciplina jurídica en nuestro ámbito nacional, de los cuales a mi juicio los más importantes a considerar son los nuevos paradigmas que se desprenden de los cambios constitucionales, convencionales, legislativos y jurisprudenciales con impacto en los campos y resultados siguientes, entre otros: 1. La distribución de competencias en el ámbito federal de gobierno; 2. La ordenación constitucional de la economía; 3. La actualización del tratado de libre comercio con Estados Unidos y Canadá; 4. El nuevo modelo de control difuso ex officio de constitucionalidad y convencionalidad; 5 . El bloque y parámetro de constitucionalidad en materia de derechos humanos; 6. Los juicios orales; 7. El proceso penal acusatorio; 8. Los 


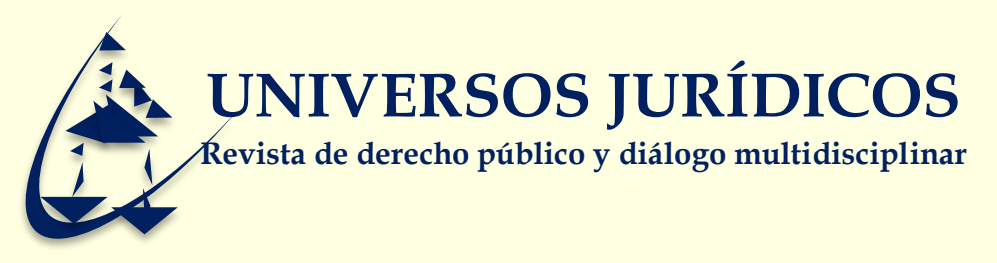

mecanismos alternativos de solución de controversias; 9. La judicialización de la justicia laboral; 10. El conjunto de reformas constitucionales derivadas de la alternancia reciente en el Ejecutivo Federal y la nueva mayoría parlamentaria.

La Corte Interamericana de Derecho Humanos ha publicado y actualizado 23 cuadernillos de jurisprudencia sobre otros tantos temas de actualidad en el mundo jurídico interamericano -criterios más importantes aún dado su carácter vinculante para todos los jueces y autoridades mexicanos-: 1 . Pena de muerte; 2. Migración; 3. Desplazamiento; 4. Género; 5. Niñas/os y adolescentes; 6. Desaparición forzada; 7. Control de Convencionalidad; 8. Libertad personal; 9. Personas privadas de libertad; 10. Integridad personal; 11. Pueblos indígenas; 12. Debido proceso; 13. Protección judicial; 14. Igualdad y no discriminación; 15. Justicia transicional; 16. Libertad de pensamiento; 17. Derecho Internacional; 18. EI Salvador; 19. Personas LGTBI; 20. Derechos Políticos; 21. Derecho a la vida; 22. DESCA; 23. Corrupción y DDHH.

El impacto de estos cambios -constitucionales, convencionales y jurisprudenciales, sobre todo- influye tanto en la Teoría del Derecho como en la Teoría de la Constitución, en la Teoría del Proceso, en la Teoría de la Prueba y en la Teoría de la Argumentación Jurídica, por lo menos. Aunque tampoco es posible desconocer que en ocasiones puede haber una retroalimentación en sentido inverso, es decir, de la teoría al derecho positivo y su interpretación.

Esto trae como consecuencia inmediata que por cuanto a la técnica de investigación documental, necesariamente deban ser estudiadas las siguientes fuentes, independientemente del objeto específico de estudio, cuando sea necesario a juicio del investigador jurídico, después de analizar su pertinencia: 1) 


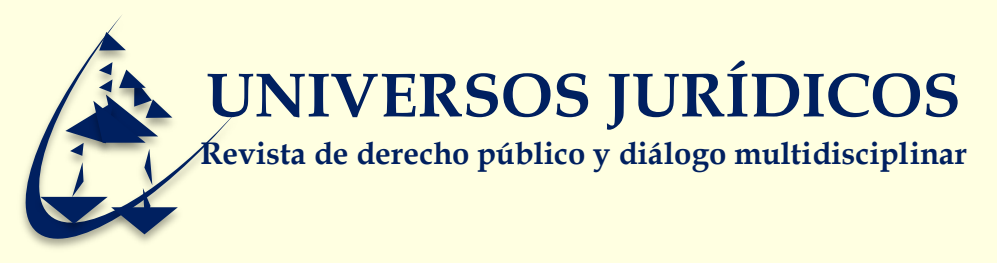

La Constitución General de la República y las de las entidades federativas, particularmente la de la Ciudad de México; 2) El nuevo bloque de constitucionalidad en materia de derecho humanos; 3) Los nuevos tratados internacionales suscritos por México con aprobación del Senado Mexicano; 4) Las leyes, reglamentos y demás disposiciones de carácter general y obligatorio federales y locales; 5) La jurisprudencia nacional e internacional, en este caso tanto la vinculante como la orientadora; 6) La doctrina nacional e internacional. EI análisis de un tema tiene que ser integral a la luz de todas estas fuentes de información y de creación o actualización de doctrina jurídica.

Como ya lo expuse desde el inicio, es frecuente el error de considerar que la disciplina jurídica tiene un carácter científico en sí misma, independientemente del método y de la metodología aplicada para descubrir o confirmar proposiciones respecto a su materia de estudio. Pero, igualmente -como también ya se ha dicho, tal vez por el carácter vinculante de las disposiciones jurídicas, es frecuente dotar a la Ciencia Jurídica de una supuesta autonomía de la que desde luego carece.

Por una parte, el método y la metodología son tan indispensables en las ciencias sociales como en las ciencias naturales o en las ciencias llamadas exactas, formales o factuales, por lo que en los estudios de Derecho no puede uno desentenderse de dichas pautas para la investigación. De la misma manera que tampoco puede, el estudioso del Derecho, aislarse y eludir los conocimientos y proposiciones de las demás disciplinas. En el estudio del Derecho como en todas las demás ciencias el enfoque multi, inter y transdisciplinario es ineludible.

La vecindad o colindancia de cualquier reporte de investigación de carácter jurídico -llámese contrato, demanda, ley, sentencia, libro, artículo o con cualquier 


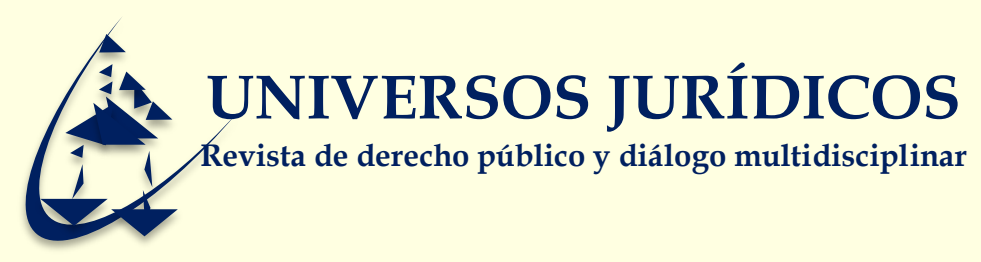

otra denominación- con otras áreas del conocimiento, no puede, de una parte, insisto, eludir éstas, de la misma manera que, de otra parte, siempre tendrá como característica propia la de conducir más tarde o temprano a una disposición vinculante inmediata o mediata, individual (contrato, sentencia) o colectiva (ley, jurisprudencia obligatoria).

Por ejemplo, aunque la verdad jurídica se apoye en la verdad histórica sobre hechos determinados puede llegar a suceder que ambas verdades no coincidan; sin embargo, será la verdad jurídica la que una vez desahogados los procedimientos de rigor tenga fuerza vinculante. Pero esto no significa que los hechos históricos o económicos o políticos $u$ otros de la realidad regulada, por regular o de las conductas por sancionar, no sean relevantes para el investigador jurídico.

En el caso de la investigación jurídica vinculada a la función legislativa me importa destacar la necesaria utilización de por lo menos dos enfoques que muestran en sí mismos la importancia de su vinculación a los estudios legislativos: el enfoque de políticas públicas ${ }^{2}$ y el de planeación prospectiva ${ }^{3}$.

\section{El reporte de la investigación}

Hasta hace relativamente poco tiempo era frecuente encontrar libros para la redacción de tesis profesionales, particularmente de la licenciatura en Derecho.

\footnotetext{
${ }^{2}$ Consultar: Análisis de Políticas Públicas, Evaluación de políticas públicas o bien Diseño de Políticas Públicas, obras cuya referencia se precisa al final de este trabajo.

${ }^{3}$ Consultar: Un modelo general de planificación, El futuro a debate, La relación entre plan nacional y planes regionales de desarrollo o bien Breve historia del futuro, cuyos datos bibliográficos también se precisan al final.
}

UNIVERSIDAD VERACRUZANA.

Instituto de Investigaciones Jurídicas

http://universosjuridicos.uv.mx/index.php/univerjuridicos/index

Xalapa, Veracruz, México 


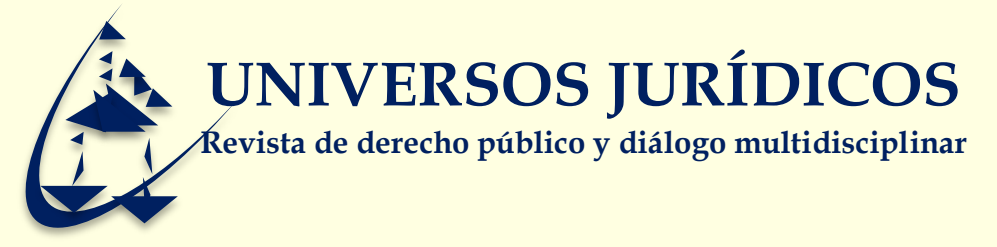

Por las razones expuestas es un tipo de bibliografía cada vez menos frecuente pero que nunca perderá su utilidad. Los libros y folletos con formularios para la redacción de contratos o para la presentación de demandas y otro tipo de escritos para comparecer ante tribunales pueden ser útiles para conocer las formalidades que imponen el trámite y la substanciación -campo minado que deben atravesar por igual, aunque con diferente suerte, el litigante y el juzgador ${ }^{4}$, pero no sustituyen el trabajo de investigación que entraña la fundamentación y motivación de dichos documentos en el caso específico.

Por fortuna, cada día son más frecuentes los libros dedicados al estudio de la redacción de sentencias y de iniciativas de ley o su dictamen. Me congratulo de contribuir a este respecto, en materia legislativa, con algunas de mis investigaciones publicadas, sobre todo una reciente -Técnica legislativa, control parlamentario y gobiernos de coalición (Castellanos, 2018)- de la cual preparo una nueva edición ${ }^{5}$. Por fortuna, también, los reglamentos de ambas cámaras legislativas federales empiezan a ocuparse de una incipiente metodología para la redacción de leyes y su dictamen, pero sin llegar a la extensión de modalidades que en este artículo se ha empezado a analizar. En el caso de redacción de artículos para revistas o libros colectivos, el formato del presente sintetiza los lineamientos editoriales de varias instituciones de cuyos comités editoriales formo parte.

\footnotetext{
${ }^{4}$ Aunque alguien pueda considerar más sencillo y rápido solicitar vía Whatsapp, en cualquier grupo de colegas abogados, copia de una demanda o recurso presentado sobre el mismo tema, pero esto tampoco exime de la indispensable investigación para defender el caso concreto; o bien buscar en cualquier base de datos judicial una sentencia sobre un caso parecido, pero siempre tomando la misma prevención.

${ }^{5}$ En un afán didáctico he propuesta uno serie de decálogos legislativos cuyo número y denominación he ampliado a partir de la experiencia práctica y las necesidades docentes.
}

\section{UNIVERSIDAD VERACRUZANA.}

Instituto de Investigaciones Jurídicas

http://universosjuridicos.uv.mx/index.php/univerjuridicos/index

Xalapa, Veracruz, México 


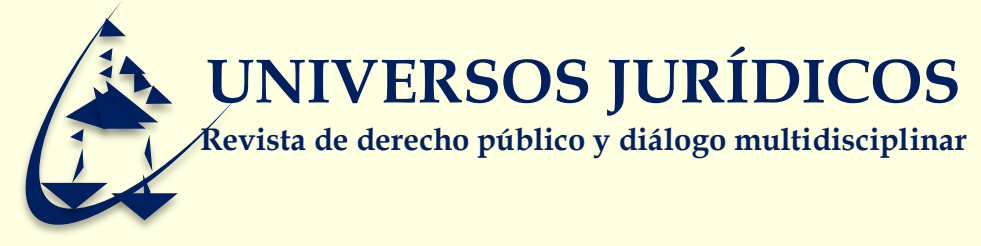

Ciertamente en cualquier papelería se puede comprar un formato de contrato de arrendamiento, pero solo después de una investigación exploratoria al menos -empezando por leer el formato adquirido- se puede concluir si es aplicable al caso concreto. En Internet es posible encontrar muchos contratos cuyas partes contratantes se encuentren en diversos países y estén sujetas a diversos ordenamientos jurídicos nacionales e internacionales, pero solo después de una investigación integral (exploratoria, descriptiva y explicativa) será posible concluir si es aplicable al caso concreto.

Me parece que con los datos metodológicos reseñados hasta aquí es posible que el investigador jurídico pueda identificar los elementos básicos de su investigación y su contexto, para determinar su amplitud y adoptar las herramientas de investigación más pertinentes, las cuales solo tienen como límites la imaginación y la disponibilidad de recursos. Como también ya lo he mencionado, deberá ajustarse a la normatividad aplicable a cada modalidad de reporte -contrato, demanda, sentencia, ley, libro, artículo, tesis-, según el ámbito institucional en el que el investigador se desempeñe o actúe -tribunal, órgano legislativo, universidad, centro de investigación, editorial-.

La hipótesis, en un asunto judicial, es la teoría del caso a probar, en tanto que en uno legislativo es la pregunta sobre la mejor manera u óptima de regular conductas mediante un ordenamiento normativo o su actualización. Las tesis de grado, los libros y los artículos, habitualmente describen el estado del arte en el campo objeto de su estudio; en ocasiones, cuando se hacen propuestas para su mejora o cambio, ésta es la materia de prueba y argumentación.

\section{UNIVERSIDAD VERACRUZANA.}




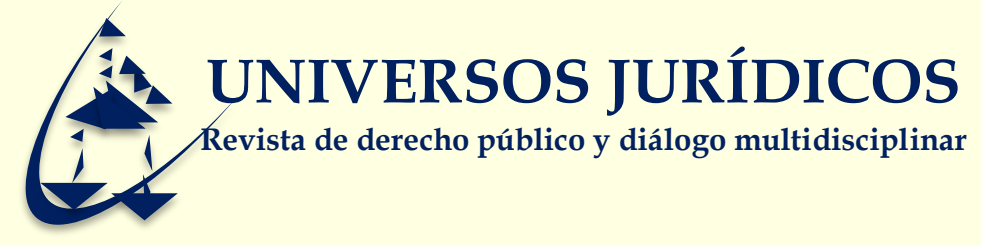

La identificación del marco teórico entraña precisar la teoría o conjunto de teorías jurídicas -pero también de otras disciplinas fácticas o formales cuando sea necesario- que explican o ayudan a explicar el fenómeno objeto de estudio. En el acápite anterior he mencionado algunas corrientes o temas -convencionales, constitucionales, legislativas, jurisprudenciales, doctrinales-, cuyos cambios implican la refutación o la adhesión por parte del investigador jurídico para llegar a nuevas conclusiones o fortalecer las existentes.

En cualquier caso, como lo señala Marc Bloch (Bloch, 2018, p. 47), "Los métodos con los que trataremos de medir el grado de certeza, serán los que realmente usa la investigación, hasta en el humilde y delicado detalle de sus técnicas".

\section{Conclusiones}

Lo antes expuesto nunca debe intimidar al investigador jurídico, sino que por el contrario lo debe motivar a conocer cada vez mejor la metodología de investigación para practicarla, dominarla y mejorarla. Incluso, el abogado litigante o el juzgador -cada uno con las restricciones que les imponen las leyes procesales-, ni remotamente agotan la sustancia de su cometido con sus habilidades y destrezas en materia de trámite y sustanciación, puesto que su objetivo principal es probar o refutar la teoría del caso.

Con mayor razón aún la metodología de investigación ofrece al legislador, formal o material -pues está sujeto a menos restricciones formales ${ }^{6}$ que el juzgador, por ejemplo-, una fuente inagotable de conocimiento para proponer

\footnotetext{
${ }^{6}$ Aunque normalmente prevalezca una restricción factual es que es la disciplina de voto.

UNIVERSIDAD VERACRUZANA.

Instituto de Investigaciones Jurídicas

http://universosjuridicos.uv.mx/index.php/univerjuridicos/index

Xalapa, Veracruz, México
} 


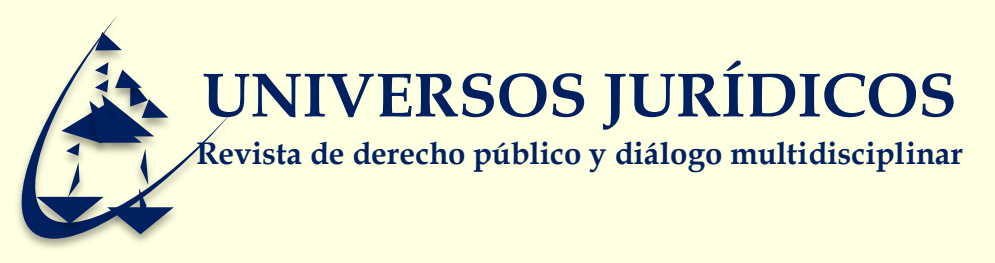

infinidad de variedades normativas y probar cuál es y por qué razones la alternativa óptima.

Finalmente, se supone que la libertad de cátedra y la libertad de investigación pudieran constituir el espacio deseable para ejercer sin límite las posibilidades que ofrecen el método y la metodología de la investigación jurídica con sus técnicas y modalidades. Pero surgen aquí otras limitantes no siempre de naturaleza diversa, por ejemplo, la disponibilidad de tiempo y de recursos o -por paradójico que parezca- la adhesión acrítica a doctrinas y teorías.

Concluyo con lo que pudiera parecer una banalidad pero que lamentablemente pudiera no serlo, al menos para los investigadores jurídicos en formación aún: la labor de estudio, de lectura, de indagación permanente, es una herramienta básica para la investigación jurídica. Solo del conocimiento adquirido principalmente de esta manera, acompañado de la observación de la realidad que aporta la experiencia práctica -también consignada en libros, reportes, dictámenes, sentencias, leyes, jurisprudencias-, será posible proponer objetos de estudio pertinentes y viables para analizarlos e innovar con base en dicho análisis.

\section{Fuentes de consulta}

Abbagnano, Nicola. (2000). Diccionario de Filosofía. Tercera edición, Segunda reimpresión. México: Fondo de Cultura Económica

Attali, Jacques. (2007). Breve historia del futuro. Barcelona, Buenos Aires, México: Paidós. 


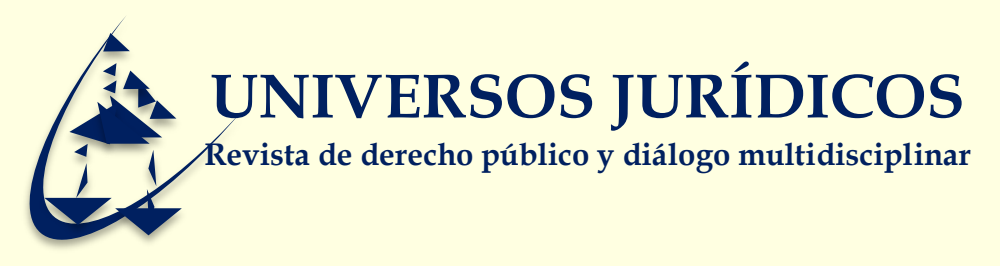

Bachelard, Gaston. (2013). La formación del espíritu científico, primera edición en español: 1948, segunda edición: 1991, duodécima reimpresión. México. Siglo veintiuno editores

Baggini, Julian. (2018). Breve historia de la verdad. Barcelona: Ático de los libros.

Báez Corona, José. (2013). Eficacia de las recomendaciones emitidas por la Comisión Nacional de Derechos Humanos en México y la reforma constitucional 2011. Una Voz Pro Persona, Año 1, No. 1, Abrilseptiembre 2013, p.31-40. http://cdigital.uv.mx/handle/123456789/36328.

Báez Corona, J. F., \& Jongitud Zamora, J. del C. (2014). La influencia de la corrupción sobre el derecho a una educación de calidad. Un estudio de correlación. Prolegómenos, 17(33), 123-142. https://doi.org/10.18359/dere.787

Bloch, Marc, (2028). Apología para la historia o el oficio de historiador. México: Fondo de Cultura Económica.

Bourdieu, Pierre, Chamboredon, Jean-Claude, Passeron, Jean-Claude. (2016). El oficio de sociólogo. Presupuestos epistemológicos, México: Siglo veintiuno editores.

Bunge, Mario. (1973). La ciencia, su método y su filosofía. Buenos Aires: Ediciones Siglo Veinte. , (2014). Ciencia, técnica y desarrollo, primera edición, siglo veinte, Buenos Aires 1980, primera edición mexicana: 2014, México. 


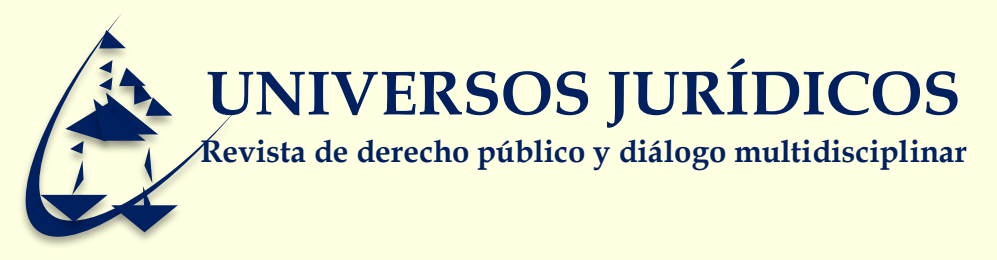

................,Epistemología, siglo veintiuno editores. México: Siglo veintiuno editores.

Carrillo Landeros, Ramiro. (1991). Metodología y administración. México: Editorial Limusa.

Castellanos Hernández, Eduardo de Jesús. (2018). Técnica legislativa, control parlamentario y gobiernos de coalición, México: Editorial Flores.

(1991). La relación entre plan nacional y planes regionales de desarrollo, México: Universidad Autónoma Benito Juárez de Oaxaca.

Diccionario de la Lengua Española. (1992). Vigésima primera edición, Tomos I y II, Madrid: Real Academia Española.

Dror, Yehezkel, Un modelo general de planificación (1980) Facultad de Ciencias Políticas y Sociales, México: UNAM.

Farina, Mario V., 1976. Diagramas de flujo, México: Editorial Diana.

Festinger, Leon, Katz, Daniel, Les méthodes de recherche dans les sciences sociales, tomes 1 et 2, Presses Universitaires de France, Paris, 1974.

Foucault, Michel. (2017). El coraje de la verdad. El gobierno de sí y de los otros, tomo II. México: Fondo de Cultura Económica.

........, (2016) El orden del discurso. México: Fábula tusQuests Editores

Franco Corzo, Julio. (2013). Diseño de políticas públicas, México: IEXE Editorial. 


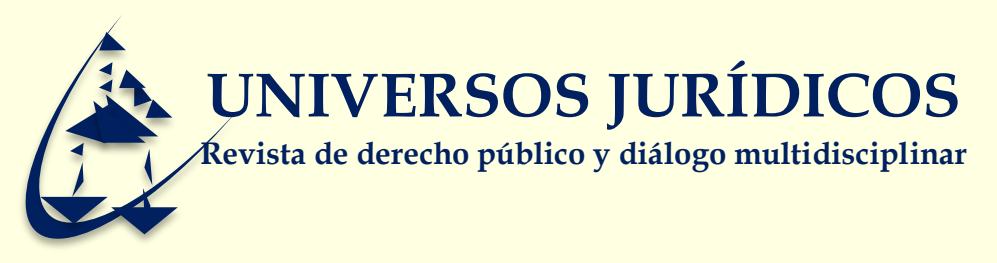

García Laguardia, Jorge Mario, Luján Muñoz, Jorge. (1980). Guía de técnicas de investigación y cuaderno de trabajo. doceava edición. México: Publicaciones Sur S. A.

Harguindéguy, Jean-Baptiste. (2015). Análisis de políticas públicas, Madrid: Tecnos.

Kuhn, Thomas S. (2015). La estructura de las revoluciones científicas, México: Fondo de Cultura Económica

Miklos, Tomás, Arroyo, Margarita (Coordinadores), (2016). El futuro a debate, México: Limusa.

Montaño, Agustín. (1977). Iniciación al método del camino crítico, tercera edición: agosto 1972, cuarta reimpresión: enero 1977, México: Editorial Trillas.

Muñoz Rocha, Carlos I. (2016). Metodología de la Investigación, primera edición: junio de 2015, primera reimpresión: marzo de, México: Oxford University Press.

Padua, Jorge. (1996). Técnicas de investigación aplicadas a las ciencias sociales, Sexta reimpresión, México: El Colegio de México, Fondo de Cultura Económica.

Pardinas, Felipe. (1973). Metodología y técnicas de investigación en ciencias sociales. Introducción elemental. décimo primera edición, octubre, México: Siglo veintiuno editores. 


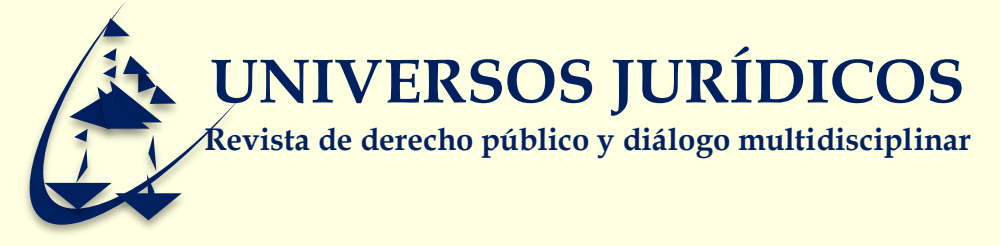

Popper, Karl. (2005). El mito del marco común. En defensa de la ciencia y la racionalidad. México: Paidós.

Salcedo, Roberto. (2011). Evaluación de políticas públicas. México: Siglo veintiuno editores.

Weber, Max. (1985). El político y el científico. México: Premiá editora de libros. 